\title{
STUDIO DELL'ATTIVITÀ DI ANALOGHI CICLICI ISOTIOSEMICARBAZONICI NEI CONFRONTI DI ISOLATI CLINICI DI ASPERGILLUS SPP.
}

Chisu L. ${ }^{a}$, Cardia M.C. ${ }^{b}$, Borgna R. ${ }^{a}$, Saddi M. ${ }^{a}$, Magnapane S. Soru A. ${ }^{\text {, }}$, Cabitza D. ${ }^{a}$, Maccioni D. ${ }^{a}$, Saddi B. ', Maccioni E. De Logu A. ${ }^{a}$

aipartimento di Scienze e tecnologie Biomediche, Sezione di Microbiologia Medica, Università di Cagliari, Viale Sant'lgnazio 38, 09123 Cagliari 'Dipartimento Farmaco Chimico Tecnologico, Università di Cagliari

Laboratorio di Analisi Ospedale SS. Trinità, Cagliari

Le infezioni sostenute dalle muffe del genere Aspergillus costituiscono la principale causa di morbilità e mortalità nei pazienti trapiantati.

L'aspergillosi invasiva rappresenta una importante causa di morte negli individui immunocompromessi.

Si tratta di un'infezione nosocomiale che colpisce i soggetti in fase di neutropenia prolungata.

La prognosi è quasi sempre infausta con un rischio di morte superiore all' $80 \%$.

Il trattamento di tali micosi nei pazienti immunodepressi si basa essenzialmente sull'uso dell'amfotericina B (AmB) e dell'itraconazolo.

La nefrotossicità rappresenta l'effetto collaterale più frequente nel corso di terapia con $\mathrm{AmB}$ ed è aumentata in caso di soministrazione contemporanea di altri farmaci nefrotossici (ciclosporina).

Sono disponibili formulazioni lipidiche di AmB che presentano una minore nefrotossicità, ma i costi elevati ne limitano l'utilizzo in terapia.

L'assunzione di itraconazolo per via orale può alleviare la sintomatologia dell'aspergilloma, ma raramente è in grado di eliminare il micete dalle cavità.

Gli effetti collaterali dell'itraconazolo possono presentarsi come risultato delle interazioni con molti altri farmaci.

È dunque necessario approfondire lo studio di nuove molecole dotate di bassa tossicità e capaci di adeguarsi alle alterate condizioni fisiologiche di questi pazienti.

Abbiamo valutato l'attività antifungina di nuovi analoghi ciclici isotiosemicarbazonici.

Sono stati utilizzati ceppi di $A$. niger e $A$. flavus isolati da campioni clinici.

La citotossicità è stata determinata mediante riduzione di 3(4,5-dimetiltiazol-2-il)-2,5difenil tetrazolio bromuro (MTT) in monostrati di cellule VERO.

I derivati isotiosemicarbazonici studiati hanno mostrato una buona attività nei confronti di Aspergillus spp.

In particolare uno di essi ha mostrato un'attività quattro volte superiore a quella dell' $A m B$ e cinque volte superiore a quella dell'itraconazolo nei confronti di A. niger.

Inoltre i bassi valori di tossicità cellulare determinano un elevato indice di selettività che supera di oltre centosettanta volte quello dell'AmB.

Quindi i derivati isotiosemicarbazonici rappresentano un valido nucleo per lo sviluppo di nuovi farmaci attivi contro Aspergillus spp. 